
ISSN-e 2619-4945

\title{
Determinantes políticos de la salud: un concepto de importancia para el profesional en salud pública. Opinión Novel
}

\section{Political determinants of health: a concept of importance for the professional in public health. Novel Opinion}

\author{
Jaime Eduardo Gómez D, Marco Tulio González A², Reinaldo Francisco Ramírez M³. \\ Recibido para publicación: Noviembre 18 de 2018 - Aprobado para publicación: Marzo 30 de 2019
}

Los factores sociales, culturales, económicos, ambientales y políticos, dentro de cualquier comunidad o población, son los que determinan las condiciones de vida, salud y bienestar de las personas. Esta relación indisoluble entre cada uno de estos factores condicionantes, y el estado de salud de los individuos, es lo que comúnmente se conoce como determinante social de la salud $(1,2)$. La dinámica con la que estos determinantes actúan sobre las poblaciones, puede dar como resultado diferencias marcadas en los estilos de vida y el nivel de bienestar que pueden gozar unos u otros.

Un determinante social de la Salud influye en el tipo de enfermedad que puede padecer una comunidad, las principales causas de muerte, la calidad de los servicios de Salud a los cuales puede acceder, además de la exposición y riesgo de adquirir cierta patología $(3,4,5,6)$. En este sentido, un determinante de la Salud, incide enormemente en la clase de vida que pueden llevar las personas; no obstante, consideramos que es el determinante político quien tiene mayor impacto y alcance sobre las condiciones de vida y salud de las mismas.

Citación (Vancouver) Gómez JE, González MT, Ramírez RF. Determinantes políticos de la salud: un concepto de importancia para el profesio-nal en salud pública. Rev Avances en Salud; 2019. (3)1:45-48. DOI: 10.21897/25394622.1751

(C) 2019. Universidad de Cordoba. Este es un artículo de acceso abierto distribuido bajo los términos de la licencia Creative Commons Attribution License, que permite el uso ilimitado, distribución y reproducción en cualquier medio, siempre que el autor original y la fuente se acreditan.

${ }^{1}$ Biólogo. M.Sc (c) en Salud Pública.

${ }^{2}$ Médico Cirujano. M.Sc (c) en Salud Pública. Correspondencia: marcogonzalez_25@hotmail.com

${ }^{3}$ Médico Cirujano. M.Sc (c) en Salud Pública. 
Gómez et. al. - Determinantes políticos de la salud: un concepto de importancia para el profesional en salud pública. Opinión Novel

De esta forma, cualquier norma, política y práctica que resulte de la interacción entre todos los sectores que afectan la salud, es considerado un determinante político (12).

El Estado, sus legisladores y las agencias ejecutivas (congresos, gobiernos, ministerios, entre otros), son ejemplos de estructuras políticas. Las elecciones, consultas y elaboración de leyes, constituyen algunos de los procesos políticos. Dentro de los productos políticos se pueden incluir las leyes, los decretos, impuestos, beneficios de seguridad social y servicios públicos, que en última instancia producirán la salud y otros resultados sociales de interés.

Asimismo, conocercómosurgen einteraccionan esos elementos, permite a los profesionales y estudiantes de la Salud pública actuar de manera efectiva dentro del ámbito político; ya sea para promover el mejoramiento y la accesibilidad a los servicios de salud o abordando las inequidades según los determinantes sociales de la Salud $(13,14,15)$.

En este sentido, son muchas las discusiones que se han generado en medio de encuentros y conferencias a nivel global, que han permitido comprender y caracterizar la dinámica con la que el determinante político afecta la Salud, y las posibles estrategias para actuar de forma directa sobre el origen de esta problemática.

Dentro de estos encuentros se pueden mencionar: la Conferencia Internacional sobre Atención Primaria de Salud celebrada en AlmaAta en 1978, la I Conferencia Internacional sobre Promoción de la Salud celebrada en Ottawa en 1986, la Conferencia Internacional de la OMS sobre acción intersectorial para la salud de 1997, y la Conferencia sobre Promoción de la Salud celebrada en Bangkok en 2005. En todas ellas, se destaca o acepta que el factor político, junto a los determinantes económicos, sociales, culturales, ambientales, conductuales y biológicos, pueden fomentar la salud o perjudicarla $(16,17)$.
De igual forma, en las conferencias mundiales celebradas en Adelaida (1988), Sundsvall (1991), Jakarta (1997), México (2000), Nairobi (2009), Helsinki (2013) y Shanghai (2016), se propuso como eje central, la promoción de acciones basadas en la elaboración de políticas públicas saludables, la creación de ambientes favorecedores de la salud, el desarrollo de habilidades personales, el refuerzo de la acción comunitaria y la reorientación de los servicios hacia la salud (18).

En conjunto, las declaraciones de todas las confe $\neg$ rencias internacionales sobre promoción de la salud de la OMS, han permitido insistir en la necesidad de transformar las estructuras económicas y políticas para superar las desigualdades sociales y al $\neg$ canzar la salud y el bienestar para todos (19). No obstante, lograr este tipo transformaciones requiere un alto compromiso político, que implica a todas las áreas del gobierno y a la participación de la ciudadanía en la priorización de los problemas, su diagnóstico, diseño de programas, implementación y evaluación (20).

La falta de voluntad política, desconocer la existencia de desigualdades y políticas para poner en marcha, los fondos públicos insuficientes, falta de capacidad para llevar a cabo proyectos multisectoriales, y la falta de apropiación del problema por parte del sector sanitario, se convierten entonces en limitantes que impiden la implementación de políticas que permitan disminuir las inequidades en salud $(21,22,23)$.

Se debe incentivar la participación de los actores políticos y autoridades públicas en el sector Salud, entendiendo la Salud Pública como campo transdisciplinar $(24,25)$, para garantizar la integralidad del enfoque social y apoyar el desarrollo de acciones, políticas públicas y estrategias, con el objetivo de establecer intervenciones efectivas que aborden los diferentes determinantes sociales de la Salud $(26,27)$. 
Gómez et. al. - Determinantes políticos de la salud: un concepto de importancia para el profesional en salud pública. Opinión Novel

Desde la formación de futuros salubristas se reconoce que:

Es muy probable que el futuro reconocimiento de la profesión venga determinado por su capacidad para influir en la adopción de políticas de salud innovadoras, en su capacidad para una acción en salud más allá de las tradicionales actividades de los servicios de salud pública... La investigación y el debate sobre políticas son imprescindible en salud pública, ya que por su naturaleza genera unos conocimientos destinados a la mejora de la salud de las poblaciones. Y aunque la ciencia puede identificar soluciones, sólo la política y sus actores pueden hacer realidad estas soluciones (28).

Se propone entonces que, el futuro salubrista e investigador en el área de la Salud pública se conviertan, no sólo en un técnico de la norma, sino, primordialmente, en un actor social que esté dispuesto, a través de los principios de cada una las Ciencias Sociales y los determinantes políticos de la Salud, a lograr hechos y acciones que generen transformación social.

\section{REFERENCIAS}

1. Ávila AM. Hacia una nueva Salud Pública: Determinantes de la Salud. Acta Médica Costarricense. 2009; 51(2): 71 73.

2. Villar AM. Factores determinantes de la salud: Importancia de la prevención. Acta Médica Peruana. 2011; 28(4): 237 241.

3. Bleda GJ. Determinantes sociales de la salud y de la enfermedad. Barataria. Revista Castellano-Manchega de Ciencias Sociales. 2005; (7): 149-160.

4. De la Torre Ugarte G, Oyola GA. Los determinantes sociales de la salud: una propuesta de variables y marcadores/ indicadores para su medición. Revista
Peruana de Epidemiología. 2014; 18(1): $1-6$.

5. Álvarez CL. Los determinantes sociales de la salud: más allá de los factores de riesgo. Revista Gerencia y Políticas de Salud. 2009; 8(17): 69-79.

6. Mejía OL. Los Determinantes Sociales de la Salud: base teórica de la salud pública. Revista Facultad Nacional de Salud Pública. 2013; 31: 28-36.

7. Lobato HS. Inequidad y desigualdad sanitaria en el marco de los determinantes sociales de la salud. Investigación y Ciencia. 2010; 18(48): 52-55.

8. Organización Mundial de la Salud. Cerrando la brecha: La política de acción sobre los Determinantes Sociales de la Salud. Documento de trabajo de la Conferencia Mundial sobre los Determinantes Sociales de la Salud. [Internet]. 2011 oct 19-21: Río de Janeiro. Recuperado de: http://www.who. int/sdhconference/discussion_paper/ Discussion-Paper-SP.pdf

9. Rodríguez AS. La desigualdad de ingreso y salud en Colombia. Perfiles Latinoamericanos. 2016; (48): 265-296.

10. Cortés A. Inequidad, pobreza y salud. Colombia Médica. 2006; 37(3): 223227.

11. LOBATO, Op.cit., p. 54.

12. Ottersen OP, Dasgupta J, Blouin C, et al. The political origins of health inequity: prospects for change. The Lancet. 2014; 383:630-67.

13. Rojas OF. Determinantes sociales de la salud y la acción política. Rev Hum Med. 2013; 13(2): 279-291.

14. Mackenbach JP. Political determinants of health. European Journal of Public 
Gómez et. al. - Determinantes políticos de la salud: un concepto de importancia para el profesional en salud pública. Opinión Novel

Health. $2014 ; 24(1): 2$.

15. Borrell CA. Las políticas para disminuir las desigualdades en salud. Gac Sanit. 2008; 22(5): 465-473.

16. Organización Mundial de la salud. Comisión sobre los determinantes sociales de la Salud. Subsanar las desigualdades en una generación: Alcanzar la equidad sanitaria actuando sobre los determinantes sociales de la Salud. Ginebra; 2008.

17. Cuba FM, Albrecht LC. A 30 años de la Carta de Ottawa. Acta Médica Peruana. 2017; 34(1): 66-67.

18. López DO, Canga AN, Agurtzane M, Pardavila BM, Belintxon M, Serrano MI., et. al. Cinco paradojas de la promoción de la salud. Gac Sanit. 2017; 31(3): 269272.

19. Mejía OL. Los Determinantes Sociales de la Salud: base teórica de la salud pública. Revista Facultad Nacional de Salud Pública. 2013; 31: 28-36.

20. BORREL, Op.cit., p. 471.

21. González RA, Mogollón O. La reforma de salud y su componente político: un análisis de factibilidad. Gac Sanit. 2002; 16(1): 39-47.
22. Hernández AI, Fernández CP. Políticas de salud y salud pública. Gac Sanit. 2007; 21 (4): 280-281.

23. Kelleher C. How exactly do politics play a part in determining health? New perspectives on an age old issue. Journal of Epidemiology and Community Health. 2002; 56(10):726.

24. González ME. La salud pública como campo transdisciplinar. Revista Facultad Nacional de Salud Pública. 2007; 25(1):71-77.

25. Libreros PL. El proceso salud enfermedad y la transdisciplinariedad. Revista Cubana de Salud Pública. 2012; 38(4):622-628.

26. ÁVILA, Op.cit., p. 73.

27. Franco GÁ. Determinación global y salud: el marco amplio de los determinantes de la salud. Revista Facultad Nacional de Salud Pública [Internet]. 2013; 31:7386.

28. Hernández AI, Fernández CP. Políticas de salud y salud pública. Gac Sanit. 2007; 21(4): 280-281. 